\title{
Article \\ Pharmacodynamics of Ceftibuten: An Assessment of an Oral Cephalosporin against Enterobacterales in a Neutropenic Murine Thigh Model
}

\author{
Maxwell J. Lasko ${ }^{1}$, Tomefa E. Asempa ${ }^{1}$ (D) and David P. Nicolau ${ }^{1,2, *}$ \\ 1 Center for Anti-Infective Research and Development, Hartford Hospital, 80 Seymour Street, \\ Hartford, CT 06102, USA; maxwell.lasko@hhchealth.org (M.J.L.); tomefa.asempa@hhchealth.org (T.E.A.) \\ 2 Division of Infectious Diseases, Hartford Hospital, Hartford, CT 06102, USA \\ * Correspondence: david.nicolau@hhchealth.org; Tel.: +1-860-972-3941; Fax: +1-860-545-3992
}

Citation: Lasko, M.J.; Asempa, T.E.; Nicolau, D.P. Pharmacodynamics of Ceftibuten: An Assessment of an Oral Cephalosporin against

Enterobacterales in a Neutropenic Murine Thigh Model. Antibiotics 2021, 10, 201. https://doi.org/10.3390/ antibiotics10020201

Academic Editors: Nicholas Dixon and Anthony William Coleman Received: 6 January 2021

Accepted: 16 February 2021

Published: 19 February 2021

Publisher's Note: MDPI stays neutral with regard to jurisdictional claims in published maps and institutional affiliations.

Copyright: (c) 2021 by the authors. Licensee MDPI, Basel, Switzerland. This article is an open access article distributed under the terms and conditions of the Creative Commons Attribution (CC BY) license (https:// creativecommons.org/licenses/by/ $4.0 /)$.

\begin{abstract}
Efforts to develop and pair novel oral $\beta$-lactamase inhibitors with existing $\beta$-lactam agents to treat extended spectrum $\beta$-lactamase (ESBL) and carbapenemase-producing Enterobacterales are gaining ground. Ceftibuten is an oral third-generation cephalosporin capable of achieving high urine concentrations; however, there are no robust data describing its pharmacodynamic profile. This study characterizes ceftibuten pharmacokinetics and pharmacodynamics in a neutropenic murine thigh infection model. Enterobacterales isolates expressing no known clinically-relevant enzymatic resistance $(n=7)$ or harboring an ESBL $(n=2)$ were evaluated. The ceftibuten minimum inhibitory concentrations (MICs) were 0.03-4 mg/L. Nine ceftibuten regimens, including a human-simulated regimen (HSR) equivalent to clinical ceftibuten doses of $300 \mathrm{mg}$ taken orally every $8 \mathrm{~h}$, were utilized to achieve various $f \mathrm{~T}>\mathrm{MIC}$. A sigmoidal $\mathrm{E}_{\max }$ model was fitted to $f \mathrm{~T}>\mathrm{MIC}$ vs. change in $\log _{10} \mathrm{CFU} /$ thigh to determine the requirements for net stasis and 1- $\log _{10} \mathrm{CFU} /$ thigh bacterial burden reduction. The growth of the $0 \mathrm{~h}$ and $24 \mathrm{~h}$ control groups was $5.97 \pm 0.37$ and $8.51 \pm 0.84 \log _{10} \mathrm{CFU} /$ thigh, respectively. Ceftibuten HSR resulted in a -0.49 to $-1.43 \log _{10} \mathrm{CFU} /$ thigh bacterial burden reduction at $24 \mathrm{~h}$ across the isolates. Stasis and $1-\log _{10} \mathrm{CFU} /$ thigh reduction were achieved with a $f \mathrm{~T}>\mathrm{MIC}$ of $39 \%$ and $67 \%$, respectively. The $f \mathrm{~T}>$ MIC targets identified can be used to guide ceftibuten dosage selection to optimize the likelihood of clinical efficacy.
\end{abstract}

Keywords: pharmacokinetics; pharmacodynamics; Gram-negative; beta-lactamase

\section{Introduction}

Gram-negative Enterobacterales that harbor extended spectrum $\beta$-lactamases (ESBLs) and carbapenemases continue to be a burden on healthcare [1-3]. ESBLs, in particular, are frequent causes of infection in both hospitalized and community-dwelling patients $[1,3]$. Few oral therapeutic options exist, resulting in the use of intravenous broad-spectrum antibiotics (i.e., carbapenems) with the potential for further resistance development $[4,5]$. Moreover, infections caused by ESBL-harboring Enterobacterales are associated with an approximately two-fold increase in the cost of hospitalization and the length of stay compared with $\beta$-lactamase naïve infections [6].

The clear need for carbapenem-sparing antibiotics that can effectively treat ESBLharboring Enterobacterales has spurred numerous oral drug development efforts over the past several years [7]. Additionally, the availability of an effective oral agent targeting these challenging organisms would provide a significant therapeutic breakthrough for patients treated outside the institutional setting. Thus, repurposing older and infrequently utilized oral $\beta$-lactam agents, such as ceftibuten, cefixime, and cefpodoxime, to be paired with investigational $\beta$-lactamase inhibitors (BLIs), has become an attractive option.

Ceftibuten has been explored as a $\beta$-lactam (BL) backbone because of its excellent bioavailability (75-90\%) and high fractional excretion in urine [8,9]. In addition, ceftibuten 
demonstrates improved in vitro stability against ESBLs compared with other oral thirdgeneration cephalosporins $[8,9]$. Ceftibuten's pharmacodynamic driver is free drug time above MIC ( $f \mathrm{~T}>\mathrm{MIC})$. The $f \mathrm{~T}>\mathrm{MIC}$ requirements that best correlate with cephalosporin efficacy vary from $40-50 \%$, as reported by the European Committee on Antimicrobial Susceptibility Testing (EUCAST) scientific committee [10], to as high as 50-70\% [11,12]. However, a formal ceftibuten pharmacodynamic assessment against clinically-relevant Enterobacterales has never been performed. Previously published studies have focused on the activity of ceftibuten in combination with BLIs, with a focus on understanding BLI exposure requirements for efficacy $[13,14]$. Thus, ceftibuten-specific pharmacodynamic profiling is needed in order to better understand the scope and potential utility of a range of exposures prior to their combination with a novel BLI. Herein, we describe a pharmacodynamic assessment of ceftibuten in a neutropenic murine thigh infection model.

\section{Results}

\subsection{Murine Pharmacokinetic Studies}

Ceftibuten single doses were well characterized using a uniform one-compartment model with first-order absorption and elimination (Figure 1). Furthermore, the pharmacokinetics of ceftibuten were relatively linear (area under the curve (AUC) $R^{2} 0.98$ ) over the single doses administered using non-compartmental analysis (Table 1). The elimination half-life ranged from 1.0 to $1.8 \mathrm{~h}$. The mean ( \pm standard deviation $(\mathrm{SD}))$ pharmacokinetic parameters, namely, volume of distribution $\left(\mathrm{V}_{\mathrm{d}}\right), 0.342 \pm 0.09(\mathrm{~L} / \mathrm{kg})$; absorption constant $\left(\mathrm{K}_{\mathrm{a}}\right), 5.45 \pm 2.46(1 / \mathrm{h})$; and elimination constant $\left(\mathrm{K}_{\mathrm{e}}\right), 0.60 \pm 0.17(1 / \mathrm{h})$, were used to simulate ceftibuten regimens and obtain concentration-time profiles. Comparisons of the $\% f \mathrm{~T}>\mathrm{MIC}$ values achieved with ceftibuten at MICs ranging between 0.03 and $4 \mathrm{mg} / \mathrm{L}$, as well as the area under the concentration-time curve from 0 to $24 \mathrm{~h}$ for the free, unbound fraction of the drug $\left(f \mathrm{AUC}_{0-24}\right)$, in mice receiving the selected regimens are presented in Table 2.

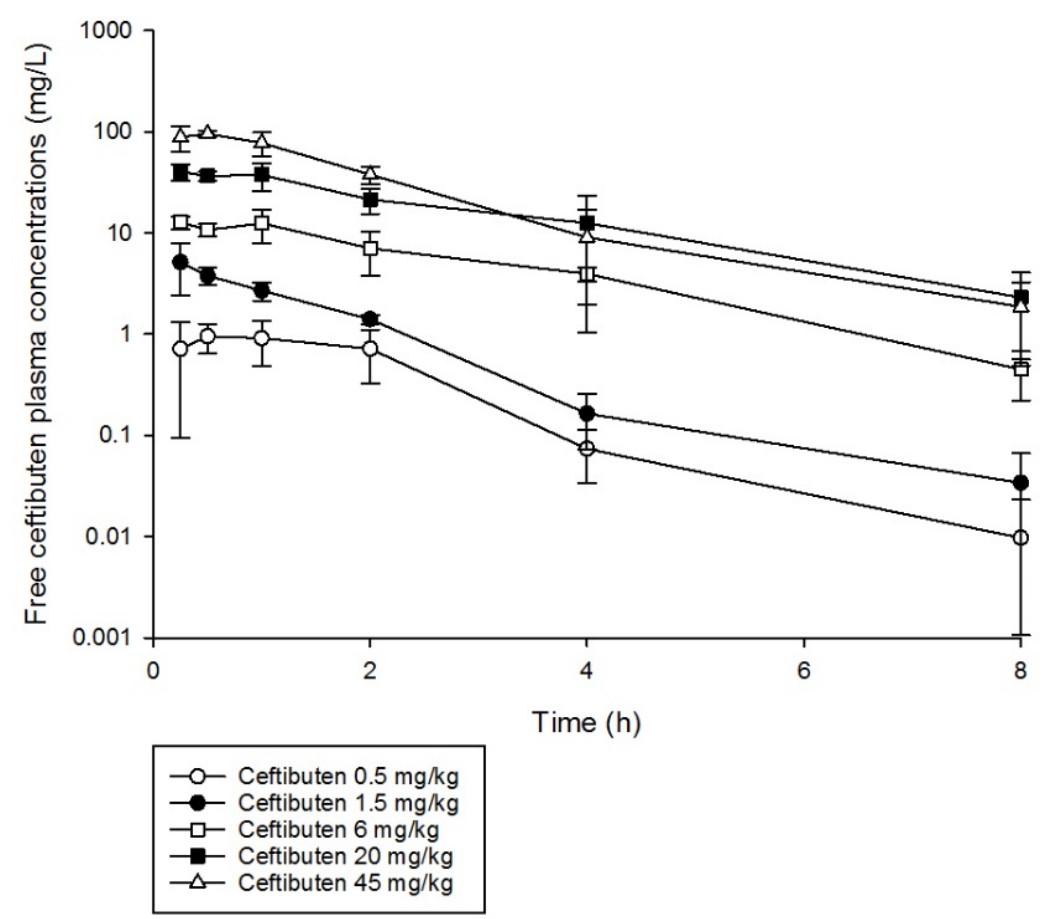

Figure 1. Ceftibuten free plasma concentration-time profiles in a neutropenic mouse thigh infection model following the administration of single subcutaneous doses $(0.5-45 \mathrm{mg} / \mathrm{kg})$. 
Table 1. Comparison of single dose ceftibuten regimens in mice using non-compartmental analysis.

\begin{tabular}{ccccc}
\hline Single Dose Regimen & $\boldsymbol{\lambda}_{\mathbf{1 / 2}}(\mathbf{h})$ & $f$ Cmax & $f$ AUC $_{\mathbf{0 - 8}}$ & $\mathbf{R}^{\mathbf{2}}$ \\
\hline $0.5 \mathrm{mg} / \mathrm{kg}$ & 1.0 & 1.0 & 2.3 & 0.90 \\
$1.5 \mathrm{mg} / \mathrm{kg}$ & 1.2 & 5.1 & 6.8 & 0.92 \\
$6 \mathrm{mg} / \mathrm{kg}$ & 1.5 & 12.8 & 37.0 & 0.98 \\
$20 \mathrm{mg} / \mathrm{kg}$ & 1.8 & 40.4 & 119.3 & 0.99 \\
$45 \mathrm{mg} / \mathrm{kg}$ & 1.4 & 95.6 & 191.0 & 0.97 \\
\hline
\end{tabular}

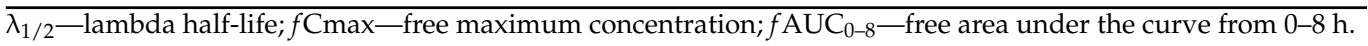

Table 2. Comparison of the $\% f \mathrm{~T}>\mathrm{MIC}$ values achieved with ceftibuten regimens at different MICs in humans and the murine thigh infection model.

\begin{tabular}{|c|c|c|c|c|c|c|c|c|c|c|}
\hline \multirow{2}{*}{ Dosing Regimen } & \multicolumn{8}{|c|}{$\% f \mathrm{~T}>\mathrm{MIC}(\mathrm{mg} / \mathrm{L})$} & \multirow{2}{*}{$f \mathrm{AUC}_{0-24}$} & \multirow{2}{*}{$\mathrm{C}_{\max }$} \\
\hline & 0.03 & 0.06 & 0.125 & 0.25 & 0.5 & 1 & 2 & 4 & & \\
\hline $\begin{array}{l}\text { CTB } 300 \text { mg q8 h } \\
\text { (human) [15] }\end{array}$ & $100 \%$ & $100 \%$ & $100 \%$ & $100 \%$ & $100 \%$ & $91 \%$ & $59 \%$ & $0 \%$ & 56.2 & 3.75 \\
\hline $\begin{array}{c}\text { CTB } 300 \text { mg q8 h HSR } \\
\text { (murine) }\end{array}$ & $100 \%$ & $100 \%$ & $100 \%$ & $100 \%$ & $100 \%$ & $91 \%$ & $50 \%$ & $0 \%$ & 50.1 & 3.6 \\
\hline $0.5 \mathrm{mg} / \mathrm{kg}$ single dose & $24 \%$ & $22 \%$ & $17 \%$ & $12 \%$ & $7 \%$ & $0 \%$ & $0 \%$ & $0 \%$ & 2.1 & 0.97 \\
\hline $3 \mathrm{mg} / \mathrm{kg}$ single dose & $37 \%$ & $35 \%$ & $30 \%$ & $25 \%$ & $20 \%$ & $15 \%$ & $10 \%$ & $4 \%$ & 12.8 & 5.8 \\
\hline $0.5 \mathrm{mg} / \mathrm{kg} \mathrm{q} 8 \mathrm{~h}$ & $71 \%$ & $66 \%$ & $51 \%$ & $36 \%$ & $21 \%$ & $0 \%$ & $0 \%$ & $0 \%$ & 6.4 & 0.98 \\
\hline $1 \mathrm{mg} / \mathrm{kg} \mathrm{q} 6 \mathrm{~h}$ & $100 \%$ & $100 \%$ & $90 \%$ & $70 \%$ & $48 \%$ & $28 \%$ & $1 \%$ & $0 \%$ & 16.89 & 2.02 \\
\hline $6 \mathrm{mg} / \mathrm{kg} \mathrm{q} 8 \mathrm{~h}$ & $100 \%$ & $100 \%$ & $100 \%$ & $91 \%$ & $76 \%$ & $61 \%$ & $45 \%$ & $30 \%$ & 76.4 & 11.8 \\
\hline $20 \mathrm{mg} / \mathrm{kg} \mathrm{q} 6 \mathrm{~h}$ & $100 \%$ & $100 \%$ & $100 \%$ & $100 \%$ & $100 \%$ & $100 \%$ & $96 \%$ & $76 \%$ & 337.8 & 40.3 \\
\hline $20 \mathrm{mg} / \mathrm{kg} \mathrm{q} 4 \mathrm{~h}$ & $100 \%$ & $100 \%$ & $100 \%$ & $100 \%$ & $100 \%$ & $100 \%$ & $100 \%$ & $100 \%$ & 500.7 & 43.8 \\
\hline $45 \mathrm{mg} / \mathrm{kg} \mathrm{q} 3 \mathrm{~h}$ & $100 \%$ & $100 \%$ & $100 \%$ & $100 \%$ & $100 \%$ & $100 \%$ & $100 \%$ & $100 \%$ & 1487.6 & 108.7 \\
\hline
\end{tabular}

$f \mathrm{~T}>\mathrm{MIC}$ - free time above the MIC; $f \mathrm{AUC}_{0-24}$ - free area under the curve for a 24-h period; $\mathrm{C}_{\max }$ - maximum concentration; HSR — humansimulated regimen.

\subsection{Pharmacodynamic Studies}

The average growth ( \pm standard deviation) of the $0 \mathrm{~h}$ and $24 \mathrm{~h}$ control groups were $5.97 \pm 0.37 \log _{10} \mathrm{CFU} /$ thigh and $8.51 \pm 0.84 \log _{10} \mathrm{CFU} /$ thigh, respectively (Figure 2). The administration of ceftibuten HSR resulted in bacterial reductions in all isolates (range: -0.49 to $1.43 \log _{10} \mathrm{CFU} /$ thigh), and five of the nine isolates achieved a $1-\log _{10} \mathrm{CFU} /$ thigh reduction. The composite exposure-response relationship for ceftibuten against individual isolates is depicted in Table 3. Reflecting the strain variability among these clinical isolates, the exposure-response relationships for the isolates were also variable, based on the coefficient of determination (average $\mathrm{R}^{2}=0.79$, range: 0.39 to 0.92 ); however, the majority were relatively robust $\left(R^{2}>0.70\right)$. Using these sigmoidal fits, three of the nine isolates did not achieve a $1-\log _{10} \mathrm{CFU} /$ thigh reduction, while one isolate did not achieve stasis. The $f \mathrm{~T}>\mathrm{MIC}$ corresponding to the stasis and $1-\log _{10}$ reduction for each individual isolate are provided in Table 3. On average, the $f \mathrm{~T}>\mathrm{MIC}$ required for the bacteriostasis and 1- $\log _{10}$ reduction against the five $E$. coli isolates evaluated was $34 \%$ and $61 \%$, respectively. A higher $f \mathrm{~T}>\mathrm{MIC}$ threshold for the stasis $(44 \%)$ and $1-\log _{10}$ reduction $(77 \%)$ was observed against the four K. pneumoniae isolates evaluated. The aggregate static targets for wild-type isolates were similar to the ESBL-harboring isolates ( $f \mathrm{~T}>\mathrm{MIC} 32 \% \mathrm{vs.} 31 \%$ ). Based on the composite exposure-response data from all isolates (Figure 3), the ceftibuten $f \mathrm{~T}>$ MIC values associated with the static and $1-\log _{10}$ targets for these nine isolates were $39 \%$ and $67 \%$, respectively. 


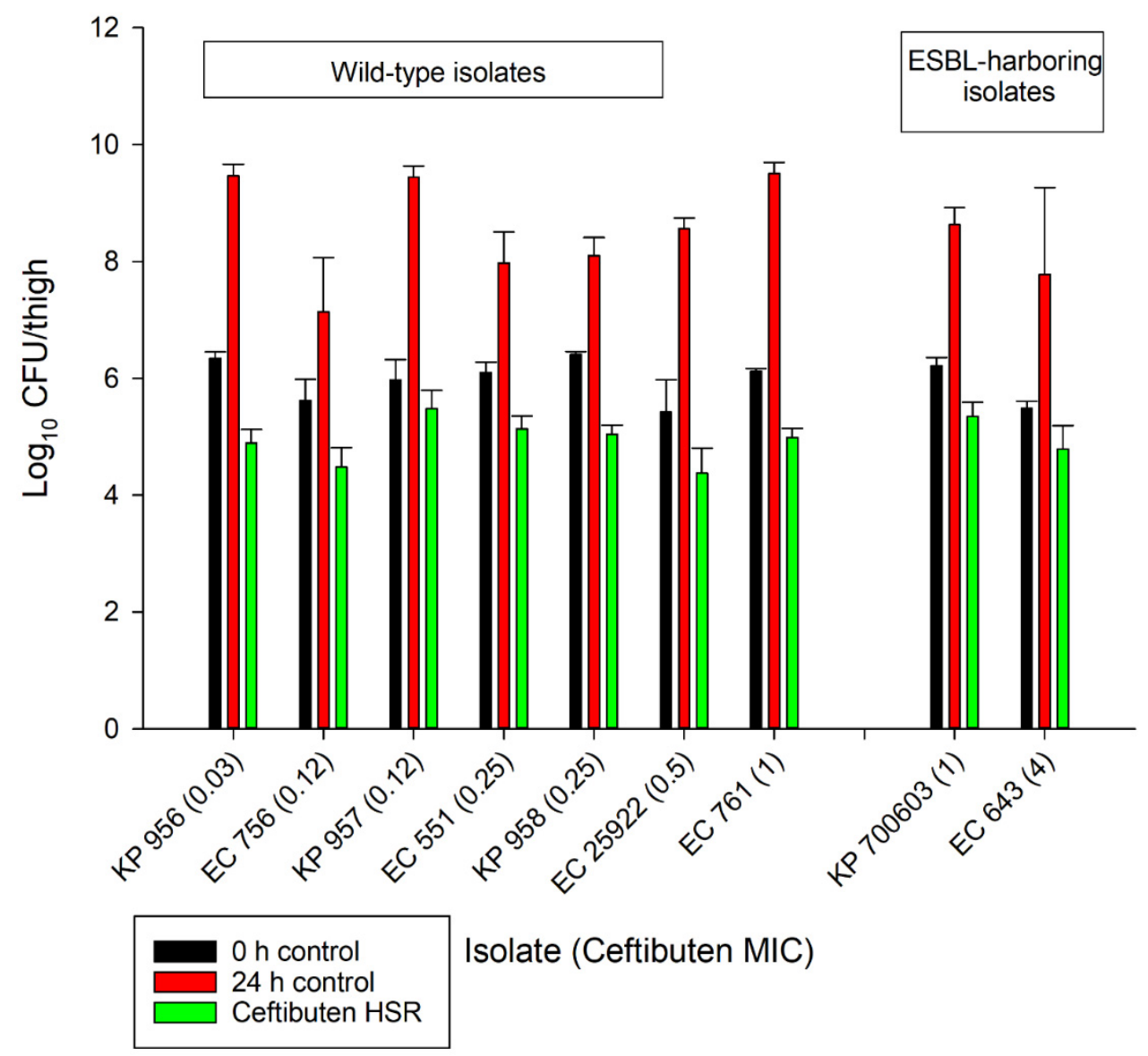

Figure 2. In vivo antibacterial activity of the ceftibuten human-simulated regimen (300 mg taken orally every $8 \mathrm{~h}$ ) compared to the $0 \mathrm{~h}$ and $24 \mathrm{~h}$ control groups.

Table 3. $\% \mathrm{fT}>\mathrm{MIC}$ pharmacodynamic targets for ceftibuten against individual isolates and the aggregate composite.

\begin{tabular}{cccc}
\hline Isolate & \multicolumn{2}{c}{ Ceftibuten $\% f$ T $>$ MIC Required to Achieve } & \multirow{2}{*}{$\mathbf{R}^{\mathbf{2}}$} \\
\cline { 2 - 3 }$(\mathbf{M I C}(\mathbf{m g} / \mathbf{L}))$ & Stasis & 1-log Reduction & 0.90 \\
\hline KP 956 (0.03) & 46 & 68 & 0.72 \\
EC 756 (0.125) & 17 & 35 & 0.74 \\
KP 957 (0.125) & NA & NA & 0.85 \\
EC 551 (0.25) & 35 & NA & 0.92 \\
KP 958 (0.25) & 40 & 59 & 0.78 \\
EC 25922 (0.5) & 26 & 56 & 0.9 \\
EC 761 (1) & 17 & 31 & 0.89 \\
KP 700603 (1) & 44 & NA & 0.39 \\
EC 643 (4) & 11 & 69 & - \\
Median $^{1}$ & 31 & 58 & $0.74-0.9$ \\
IQR $^{1}$ & $17-41$ & $40-66$ & \\
\hline
\end{tabular}

1_calculated from isolates that achieved the target goal; NA—not achieved. 


\section{CTB PD Aggregate Composite}

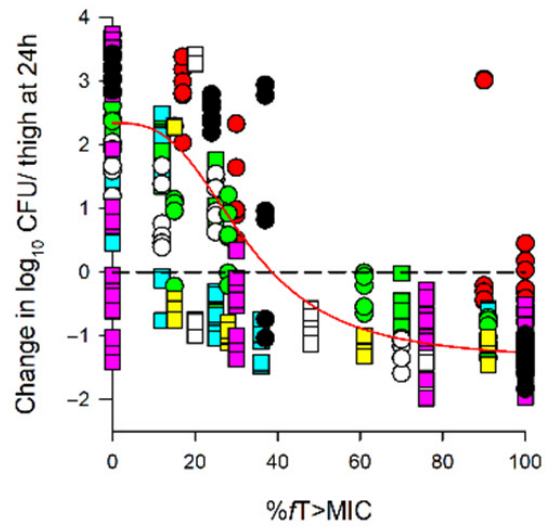

CTB PD Wild-Type Composite
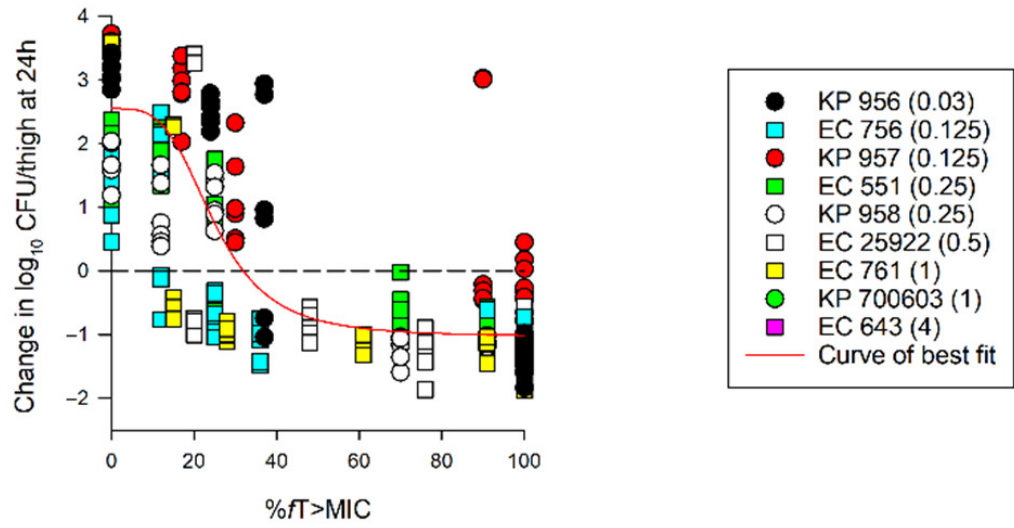

CTB PD ESBL-Harboring Isolate Composite

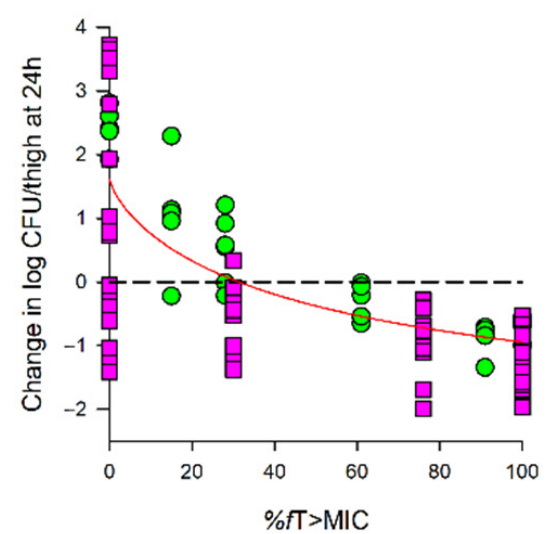

Figure 3. Sigmoid $E_{\max }$ curves depicting $\% f T>M I C$ and change in $\log _{10} \mathrm{CFU} /$ thigh for all isolates (aggregate composite), wild-type isolates, and extended spectrum $\beta$-lactamase (ESBL)harboring isolates. Each dot represents the $\log _{10} \mathrm{CFU} /$ thigh for each bacterial strain (ceftibuten MIC) per regimen. $\mathrm{KP}-$ Klebsiella pneumoniae; EC-Escherichia coli (EC). Aggregate composite: $\mathrm{R}^{2}=0.79, \mathrm{EC}_{50}=32.46 \%$, and $\mathrm{E}_{\max }=2.34 \log _{10} \mathrm{CFU} /$ thigh; wild-type composite: $\mathrm{R}^{2}=0.83$, $\mathrm{EC}_{50}=24.92 \%$, and $\mathrm{E}_{\max }=2.55 \log _{10} \mathrm{CFU} /$ thigh; $\mathrm{ESBL}$ composite: $\mathrm{R}^{2}=0.64, \mathrm{EC}_{50}=58.87 \%$, and $\mathrm{E}_{\max }=2.34 \log _{10} \mathrm{CFU} /$ thigh.

\section{Discussion}

The combination of novel BLIs with already-approved $\beta$-lactam agents continues to be a safe and attractive drug development strategy for combating the growing threat of 
antimicrobial resistance [6]. Although the majority of combination products in the pipeline are intravenous, a push towards the development of oral combination products to serve as a step-down option or to facilitate outpatient management is gaining momentum $[7,16]$. Characterizing the range of microbiological activity of $\beta$-lactam is imperative for understanding its potential activity against antibiotic resistant isolates when combined with a BLI. Therefore, this in vivo study sought to bridge the gap in ceftibuten pharmacodynamic knowledge and provide relevant exposure-response data against wild-type and ESBL-harboring isolates.

The ceftibuten dose-ranging experiments in this study demonstrated that a mean ceftibuten $f \mathrm{~T}>\mathrm{MIC}$ of $67 \%$ translated into a $1-\log _{10} \mathrm{CFU} /$ thigh reduction in bacterial burden. This data provide a ceftibuten-specific target, negating the extrapolation from ranges (40-70\%) determined from other cephalosporin pharmacodynamic studies [10-12]. In addition, net stasis, an adopted microbiological surrogate for clinical efficacy in urinary tract infections (UTIs), [17] was observed at a ceftibuten $f$ T > MIC of $39 \%$.

Enzymatic mechanisms of resistance continue to be a major burden on public health, and ESBL-harboring isolates are no exception. ESBL-harboring Enterobaterales isolates continue to be a contributor to patient morbidity and mortality, with incidence increasing by 53\% from 2012 to 2017 [18]. Unlike other forms of enzymatic resistance, ESBLs can commonly manifest in community and hospital-acquired infections [1]. The development of an appropriate oral BL/BLI against this infection entity is imperative. Ceftibuten is stable against narrow-spectrum ESBLs, but is readily hydrolyzed by broader spectrum variants $[8,9]$. In this study, the pharmacodynamic targets for ESBL-harboring isolates, albeit limited by the small sample size, were comparable to their wild-type counterpart, and were similar to previous findings [19]. In addition to classic dose-ranging studies, we also utilized a ceftibuten human-simulated regimen to evaluate the bactericidal activity of clinicallyrelevant ceftibuten exposure. Concordant with its clinical utility for UTIs, ceftibuten at an exposure equivalent to that of a human oral dose of $300 \mathrm{mg}$ taken orally every $8 \mathrm{~h}$ resulted in bacterial stasis in all isolates and achieved a $1-\log _{10} \mathrm{CFU} /$ thigh reduction in five isolates. This regimen can be used in conjunction with a novel $\beta$-lactamase-inhibitor to optimize exposure profiles against ESBL- and carbapenemase-harboring isolates in vivo. Further studies evaluating the therapeutic potential of ceftibuten drug combinations are warranted.

\section{Materials and Methods}

\subsection{Antimicrobial Test Agents}

Analytical grade ceftibuten (lot RCHX170002, Covalent Laboratories Private Limited, Hyderabad, India) was reconstituted and diluted to the desired dosing concentrations in a $50 \mathrm{mM}$ sodium phosphate buffer. All doses were administered subcutaneously with a final volume of $0.2 \mathrm{~mL}$.

\subsection{Isolates}

A total of nine clinical Enterobacterales isolates were obtained from the Centers for Disease Control and Food and Drug Administration Antimicrobial Resistance Isolate Bank $(n=1)$, the Antibacterial Research Leadership Group isolate bank $(n=5)$, the American Type Culture Collection $(n=2)$, and the Center for Anti-Infective Research and Development (CAIRD) isolate library $(n=1)$. Escherichia coli $(n=5)$ and Klebsiella pneumoniae $(n=4)$ isolates were selected for their ESBL-harboring and wild-type genotypes. The ceftibuten broth microdilution MICs ranged from $0.03-4 \mathrm{mg} / \mathrm{L}$. The individual isolate genotype and ceftibuten MICs are available in Table 4. Notably, one K. pneumoniae isolate (KP 956) was characterized as SHV-harboring, but displayed the phenotypic profile (ceftibuten MIC: $0.03 \mathrm{mg} / \mathrm{L}$ ) of a wild-type isolate and thus was included in the wild-type isolate analysis. All of the isolates were stored frozen at $-80^{\circ} \mathrm{C}$ in skim milk. Prior to examination, each isolate was sub-cultured twice on trypticase soy agar with $5 \%$ sheep blood (Becton Dickinson and Co., Sparks, MD, USA) and incubated at $37^{\circ} \mathrm{C}$ for $18-24 \mathrm{~h}$. 
Table 4. Genotypic and phenotypic profiles of ceftibuten against the test isolates.

\begin{tabular}{ccccc}
\hline Organism & CAIRD ID & Strain & $\begin{array}{c}\text { Known Resistance } \\
\text { Mechanism(s) }\end{array}$ & $\begin{array}{c}\text { Ceftibuten MIC } \\
\text { (mg/L) }\end{array}$ \\
\hline Klebsiella pneumoniae & 956 & ARLG 1112 & SHV & 0.03 \\
Escherichia coli & 756 & ARLG 1023 & None & 0.12 \\
Klebsiella pneumoniae & 957 & ARLG 1118 & None & 0.12 \\
Escherichia coli & 551 & CDC 0077 & None & 0.25 \\
Klebsiella pneumoniae & 958 & ARLG 1120 & None & 0.25 \\
Escherichia coli & 25922 & ATCC 25922 & None & 0.5 \\
Escherichia coli & 761 & ARLG 1050 & SHV-18, OXA-2, OKP-B-6 & 1 \\
Klebsiella pneumoniae & $700603^{*}$ & ATCC 700603 & CTX-M2 & 1 \\
Escherichia coli & $643^{*}$ & SI-LP377 & 4 \\
\hline
\end{tabular}

CAIRD—Center for Anti-Infective Research and Development; ARLG-Antibacterial Research Leadership Group; CDC—Centers for Disease Control; ATCC_-American Type Culture Collection. * ESBL-harboring isolates.

\subsection{Animals}

Specific-pathogen-free, female, CD-1 mice (20-22 g) were obtained from Charles River Laboratories, Inc. (Raleigh, NC, USA). All animals were allowed to acclimatize for $48 \mathrm{~h}$ prior to the study procedures, and were housed in groups of six animals at controlled room temperature in HEPA-filtered cages (Innovive, San Diego, CA, USA). The cages were supplemented with paper nesting material for enrichment purposes. Study rooms were maintained with diurnal cycles ( $12 \mathrm{~h}$ light $/ 12 \mathrm{~h}$ dark), and food and water were provided ad libitum. Animals were monitored three times daily for signs of morbidity and were euthanized if found moribund; tissues were harvested subsequent to euthanasia.

\subsection{Neutropenic Thigh Infection Model}

Prior to both the pharmacokinetic and in vivo efficacy studies, the animals were prepared as follows: neutropenia was induced by administering $150 \mathrm{mg} / \mathrm{kg}$ of intraperitoneal (i.p.) cyclophosphamide on day 4 and $100 \mathrm{mg} / \mathrm{kg}$ on day 1 . In addition, a predictable degree of renal impairment was produced using $5 \mathrm{mg} / \mathrm{kg}$ of uranyl nitrate administered i.p. on day 3 [20]. Bacterial suspensions of $\sim 1 \times 10^{7}$ colony forming units (CFU) $/ \mathrm{mL}$ in normal saline were used for the inoculation of both thighs (injection volume $0.1 \mathrm{~mL}$ ) $2 \mathrm{~h}$ prior to the first antibacterial dose.

\subsection{Murine Pharmacokinetic Studies}

The ceftibuten pharmacokinetic parameters were derived from single dose pharmacokinetic studies. Briefly, six mice per time point were prepared for experimentation as described above, and were then subjected to a single dose of ceftibuten $(0.5 \mathrm{mg} / \mathrm{kg}$, $1.5 \mathrm{mg} / \mathrm{kg}, 6 \mathrm{mg} / \mathrm{kg}, 20 \mathrm{mg} / \mathrm{kg}$, and $45 \mathrm{mg} / \mathrm{kg}$ ). The mice were euthanized by $\mathrm{CO}_{2}-$ asphyxiation and blood samples were obtained by cardiac puncture. Six time-points were assessed $(0.25 \mathrm{~h}, 0.5 \mathrm{~h}, 1 \mathrm{~h}, 2 \mathrm{~h}, 4 \mathrm{~h}$, and $8 \mathrm{~h})$. Blood was collected in $\mathrm{K}_{2}$ EDTA Tubes (Becton Dickinson and Co., Sparks, MD, USA) and centrifuged at 10,000 rpm for $10 \mathrm{~min}$ at $8{ }^{\circ} \mathrm{C}$. The separated plasma was stored at $-80^{\circ} \mathrm{C}$ until the total drug concentrations were determined using an ultra-performance liquid chromatography with tandem mass spectrometry method. The pharmacokinetic parameters were calculated using the mean drug concentrations from each group of mice, while the AUC was estimated using the linear-up log-down trapezoidal rule. Using the pharmacokinetic parameter estimates derived from the single dose pharmacokinetic studies, concentration-time profiles over $24 \mathrm{~h}$ were simulated to obtain $f \mathrm{~T}>$ MIC values for the respective doses administered. All pharmacokinetic analyses were performed in Phoenix WinNonlin (Pharsight Corp., Mountainview, CA, USA). The final weighting schemes were decided on by considering the Akaike information criterion and best visual fit. The averaged pharmacokinetic parameters were also used to develop a human-simulated regimen. Simulated-free ceftibuten concentrations were determined by considering the extent of murine protein binding (19.7\%) [13]. 


\subsection{Pharmacodynamic Studies}

Eight ceftibuten regimens with doses ranging from 0.5 to $45 \mathrm{mg} / \mathrm{kg}$ over a frequency of once to every $3 \mathrm{~h}$ were developed to achieve various $f \mathrm{~T}>\mathrm{MICs}$. In addition, a previously developed ceftibuten human-simulated dosing regimen (HSR) was administered to achieve plasma exposures similar to those achieved in humans following an oral dose of $300 \mathrm{mg}$ of ceftibuten every $8 \mathrm{~h}[15,21]$. During experimentation, 7 groups ( 2 control and 5 treatment groups) of 3 mice each were inoculated with the respective isolates. Two hours after thigh inoculation, one group was sacrificed at $0 \mathrm{~h}$ via $\mathrm{CO}_{2}$-asphxyation and cervical dislocation in order to determine the baseline bacterial burden. The remaining 6 groups received a subcutaneous injection of one of the following regimens for $24 \mathrm{~h}$ : ceftibuten HSR, a selected ceftibuten regimen to achieve a targeted $f \mathrm{~T}>\mathrm{MIC}$, or an injection of $0.9 \%$ normal saline (NS) given at the same frequency as the ceftibuten HSR. After $24 \mathrm{~h}$, all treatment groups were euthanized and the thighs ( $n=6 /$ group) were aseptically harvested and homogenized in NS. Each thigh was treated as an independent value. The homogenized thigh was serially diluted onto trypticase soy agar with $5 \%$ sheep blood (Becton Dickinson and Co., Sparks, MD, USA), and colonies were enumerated to determine the number of CFU per thigh after incubation overnight. The efficacy of each regimen was determined using the change in $\log _{10} \mathrm{CFU} /$ thigh from the $0 \mathrm{~h}$ control. $\log _{10}$ change in $\mathrm{CFU} /$ thigh was reported as mean $\pm \mathrm{SD}$ for ceftibuten HSR. A uniform $\mathrm{E}_{\max }$ model using the Hill equation was fitted to the $f \mathrm{~T}>\mathrm{MIC}$ vs. change in bacterial burden at $24 \mathrm{~h}$ using Phoenix WinNonlin for individual isolates. An aggregate composite model derived from the averaged $\mathrm{E}_{\max }$ model parameters of all bacterial strain data was constructed. Similarly, composite profiles for all the wild-type, ESBL-harboring, E. coli, and K. pneumonia isolates were also constructed using their respective strain data. These models were used to calculate effective stasis and $1-\log _{10}$ reduction pharmacodynamic targets for individual isolates and the aggregate composite.

\section{Conclusions}

To the best of our knowledge, this is the first study examining the pharmacodynamics of ceftibuten. In conjunction with the classic exposure-response profiles, the clinical exposure assessment using the human-simulated regimen provides a framework for the selection of ceftibuten as a partner agent in BL/BLI oral combinations against isolates frequently causing urinary tract infections. Additional studies evaluating these types of drug combinations are warranted.

Author Contributions: All authors contributed significantly to the completion of this paper in the following manner: conceptualization, D.P.N.; methodology, M.J.L., T.E.A., and D.P.N.; formal analysis, M.J.L., T.E.A., and D.P.N.; writing—original draft preparation, M.J.L.; writing—review and editing, M.J.L., T.E.A., and D.P.N.; funding acquisition, D.P.N. All authors have read and agreed to the published version of the manuscript.

Funding: This project was funded by Venatorx Pharmaceuticals, Inc. (Malvern, PA, USA), with federal funds from the National Institute of Allergy and Infectious Diseases, National Institute of Health, and Department of Health and Human Services, under contract no. HHSN272201600029C. The funders provided financial support and did not exercise control over the conduct or reporting of the research.

Institutional Review Board Statement: All animal experiments were conducted in accordance with the National Research Council of the National Academy of Sciences standards. The study protocol (\#HHC-2018-0052) was approved by the Institutional Animal Care and Use Committee of Hartford Hospital (assurance \#A3185-01).

Informed Consent Statement: Not applicable.

Data Availability Statement: Data is available upon reasonable request to corresponding author. 
Acknowledgments: We would like to recognize Alissa Padgett, Zach Fazzino, Deborah Santini, Janice Cunningham, Julio Rodriguez, Jennifer Tabor-Rennie, Rebecca Stewart, Nicole DeRosa, Ceara Wetteman, Lauren McLellan, Elizabeth Cyr, and Christian Gill from the Center for Anti-Infective Research and Development, and Lisa McLaughlin from Venatorx, for their assistance in this study.

Conflicts of Interest: D.P.N. has served as a consultant and speaker's bureau member, and has received research funding from Allergan, Cepheid, Merck, Pfizer, Wockhardt, Shionogi, Tetraphase, and Venatorx. M.J.L. and T.E.A. have no conflicts of interest to declare.

\section{References}

1. Bush, K.; Bradford, P.A. Epidemiology of $\beta$-Lactamase-Producing Pathogens. Clin. Microbiol. Rev. 2020, 33. [CrossRef] [PubMed]

2. Guh, A.Y.; Bulens, S.N.; Mu, Y.; Jacob, J.T.; Reno, J.; Scott, J.; Wilson, L.E.; Vaeth, E.; Lynfield, R.; Shaw, K.M.; et al. Epidemiology of Carbapenem-Resistant Enterobacteriaceae in 7 US Communities, 2012-2013. JAMA 2015, 314, 1479-1487. [CrossRef] [PubMed]

3. McDanel, J.; Schweizer, M.; Crabb, V.; Nelson, R.; Samore, M.; Khader, K.; Blevins, A.E.; Diekema, D.; Chiang, H.-Y.; Nair, R.; et al. Incidence of Extended-Spectrum $\beta$-Lactamase (ESBL)-Producing Escherichia coli and Klebsiella Infections in the United States: A Systematic Literature Review. Infect. Control Hosp. Epidemiol. 2017, 38, 1209-1215. [CrossRef] [PubMed]

4. Meije, Y.; Pigrau, C.; Fernández-Hidalgo, N.; Clemente, M.; Ortega, L.; Sanz, X.; Loureiro-Amigo, J.; Sierra, M.; Ayestarán, A.; Morales-Cartagena, A.; et al. Non-intravenous carbapenem-sparing antibiotics for definitive treatment of bacteraemia due to Enterobacteriaceae producing extended-spectrum $\beta$-lactamase (ESBL) or AmpC $\beta$-lactamase: A propensity score study. Int. J. Antimicrob. Agents 2019, 54, 189-196. [CrossRef] [PubMed]

5. Patel, N.; Harrington, S.; Dihmess, A.; Woo, B.; Masoud, R.; Martis, P. Clinical epidemiology of carbapenem-intermediate or -resistant Enterobacteriaceae. J. Antimicrob. Chemother. 2011, 66, 1600-1608. [CrossRef] [PubMed]

6. Lee, S.Y.; Kotapati, S.; Kuti, J.L.; Nightingale, C.H.; Nicolau, D.P. Impact of extended-spectrum beta-lactamase-producing Escherichia coli and Klebsiella species on clinical outcomes and hospital costs: A matched cohort study. Infect. Control Hosp. Epidemiol. 2006, 27, 1226-1232. [CrossRef] [PubMed]

7. Papp-Wallace, K.M. The latest advances in $\beta$-lactam/ $\beta$-lactamase inhibitor combinations for the treatment of Gram-negative bacterial infections. Expert Opin. Pharmacother. 2019, 20, 2169-2184. [CrossRef] [PubMed]

8. Owens, R.C., Jr.; Nightingale, C.H.; Nicolau, D.P. Ceftibuten: An overview. Pharmacotherapy 1997, 17, 707-720. [PubMed]

9. Stewart, A.G.; Harris, P.N.A.; Henderson, A.; Schembri, M.A.; Paterson, D.L. Oral cephalosporin and $\beta$-lactamase inhibitor combinations for ESBL-producing Enterobacteriaceae urinary tract infections. J. Antimicrob. Chemother. 2020, 75, $2384-2393$. [CrossRef] [PubMed]

10. European Society of Clincial Microbiology and Infectious Diseases. Why Do EUCAST Have No Systemic Breakpoints for Enterobacterales with Oral Cephalosporins? Version 2. EUCAST. Available online: https://www.eucast.org/fileadmin/src/ media/PDFs/EUCAST_files/Guidance_documents/Oral_ceph_breakpoints_v2_20200710.pdf (accessed on 20 December 2020).

11. Auckenthaler, R. Pharmacokinetics and pharmacodynamics of oral beta-lactam antibiotics as a two-dimensional approach to their efficacy. J. Antimicrob. Chemother. 2002, 50 (Suppl. 1), 13-17. [CrossRef] [PubMed]

12. Kuti, J.L. Optimizing antimicrobial pharmacodynamics: A guide for your stewardship program. Rev. Med. Clin. Condes 2016, 27, 615-624. [CrossRef]

13. Abdelraouf, K.; Stainton, S.M.; Nicolau, D.P. In Vivo Pharmacodynamic Profile of Ceftibuten-Clavulanate Combination against Extended-Spectrum- $\beta$-Lactamase-Producing Enterobacteriaceae in the Murine Thigh Infection Model. Antimicrob. Agents Chemother. 2019, 63. [CrossRef] [PubMed]

14. Lomovskaya, O.; Tsivkovski, R.; Nelson, K.; Rubio-Aparicio, D.; Sun, D.; Totrov, M.; Dudley, M.N. Spectrum of Beta-Lactamase Inhibition by the Cyclic Boronate QPX7728, an Ultrabroad-Spectrum Beta-Lactamase Inhibitor of Serine and Metallo-BetaLactamases: Enhancement of Activity of Multiple Antibiotics against Isogenic Strains Expressing Single Beta-Lactamases. Antimicrob. Agents Chemother. 2020, 64. [CrossRef]

15. Lin, C.; Lim, J.; Radwanski, E.; Marco, A.; Affrime, M. Pharmacokinetics and dose proportionality of ceftibuten in men. Antimicrob. Agents Chemother. 1995, 39, 359-361. [CrossRef] [PubMed]

16. World Health Organization. Target Product Profiles for Oral Therapy of Urinary Tract Infections; World Health Organization: Geneva, Switzerland, 2020.

17. Grayson, M.L.; Crowe, S.M.; McCarthy, J.S.; Mills, J.; Mouton, J.W.; Norrby, S.R.; Paterson, D.; Pfaller, M.A. Kucers' the Use of Antibiotics Sixth Edition: A Clinical Review of Antibacterial, Antifungal and Antiviral Drugs; CRC Press: Boca Raton, FL, USA, 2010.

18. Jernigan, J.A.; Hatfield, K.M.; Wolford, H.; Nelson, R.E.; Olubajo, B.; Reddy, S.C.; McCarthy, N.; Paul, P.; McDonald, L.C.; Kallen, A.; et al. Multidrug-Resistant Bacterial Infections in U.S. Hospitalized Patients, 2012-2017. N. Engl. J. Med. 2020, 382, 1309-1319. [CrossRef] [PubMed]

19. Andes, D.; Craig, W.A. Treatment of infections with ESBL-producing organisms: Pharmacokinetic and pharmacodynamic considerations. Clin. Microbiol. Infect. 2005, 11, 10-17. [CrossRef] [PubMed] 
20. Nicolau, D.P.; Onyeji, C.O.; Zhong, M.; Tessier, P.R.; Banevicius, M.A.; Nightingale, C.H. Pharmacodynamic assessment of cefprozil against Streptococcus pneumoniae: Implications for breakpoint determinations. Antimicrob. Agents Chemother. 2000, 44, 1291-1295. [CrossRef] [PubMed]

21. Avery, L.M.; Abdelraouf, K.A.; Nicolau, D.P. Assesment of the In Vivo Pharmacodynamic Profile of Ceftibuten/VNRX-7145 Combination against Serine-Beta-Lactamase-Producing Enterobacteriaceae in the Neutropeneic Murine Thigh Infection Model. Presented at ASM Microbe, San Fransisco, CA, USA, 20-24 June 2019. 